\title{
Troglostrongylus brevior in an Eurasian lynx (Lynx lynx) from Bosnia and Herzegovina
}

Amer Alić ${ }^{1}$ Donato Traversa ${ }^{2}$, Georg Gerhard Duscher ${ }^{3}$, Mirsad Kadrić ${ }^{1}$, Angela Di Cesare ${ }^{2}$ and Adnan Hodžic $3^{*^{*}}$ (I)

\begin{abstract}
Background: In the past few years the interest of the scientific community on lungworms of the genus Troglostrongylus has grown due to the increased number of unexpected cases of infections with Troglostrongylus brevior in domestic cats from Mediterranean Europe, likely due to a spill-over from wild reservoirs. Thus, there is a merit to increase our knowledge on the occurrence of this parasite in felids from European regions. The present paper describes lung lesions associated with T. brevior infection in the endangered Eurasian lynx (Lynx lynx) from Bosnia and Herzegovina.

Findings: The carcass of an illegally killed 3-year-old male Eurasian lynx was presented for necropsy at the Faculty of Veterinary Medicine of Sarajevo (Bosnia and Herzegovina). Grossly, multiple, multinodular, consolidated and firm, tan to grey areas, occupying the caudal third of caudal lung lobes, were observed. At cut section, the catarrhal fluid was draining from the airways. Larvae of T. brevior were found in tracheal scraping. The histopathological examination revealed multifocal to coalescing areas, centered on bronchi and bronchioles, and expanded alveoli filled with necrotic debris, degenerated inflammatory cells, mostly neutrophils and macrophages, and multiple cross sections of parasite larvae and thin-walled morulated eggs of lungworms. The paraffin-embedded lung samples were molecularly positive for $T$. brevior.

Conclusion: This paper describes the first record of T. brevior in the Eurasian lynx and the associated host lung pathology. Given its pathogenic potential and the lack of data on troglostrongylosis in lynx populations, the occurrence and impact of Troglostrongylus spp. on wildlife health as well as the role of L. Iynx as reservoir of infection for other felids, should be further investigated.
\end{abstract}

Keywords: Troglostrongylus brevior, Eurasian lynx, Lynx lynx, Pneumonia, Bosnia and Herzegovina

\section{Background}

The genus Troglostrongylus Vevers, 1923 (Metastrongyloidea: Crenosomatidae) encompasses four species of parasitic nematodes that have been described for the first time from the respiratory system of wild felids, i.e. Troglostrongylus troglostrongylus Vevers, 1923, T. brevior Gerichter, 1949, T. subcrenatus Railliet and Henry, 1913 and T. wilsoni Stough, 1953 [1]. Animals become infected by ingesting the infective stages in the intermediate hosts, i.e. snails and slugs, or more frequently in paratenic hosts, i.e. amphibians, birds, reptiles and rodents [1, 2]. In addition, direct transmission of

\footnotetext{
* Correspondence: adnan.hodzic@vetmeduni.ac.at

${ }^{3}$ Institute of Parasitology, Department of Pathobiology, University of

Veterinary Medicine Vienna, Veterinaerplatz 1, 1210 Vienna, Austria

Full list of author information is available at the end of the article
}

T. brevior from the queen to the kittens has recently been hypothesised [3].

Although regarded as a parasite of wild felids [1, 2, 4], in the past few years $T$. brevior has been described in domestic cats from islands of Spain [5], Italy [6, 7] and Greece [8] and from hilly and mountainous sub-Apennine areas of Italy [4, 9-12]. In these cases clinical presentation and lesions ranged from subclinical infections to severe pathologies with lifethreatening or fatal outcomes in kittens and young cats [4-6, 8-12]. Furthermore, T. brevior may contribute to more severe lung pathology when co-infections with $A$. abstrusus occur [11]. In Italy, the endangered European wildcat (Felis silvestris silvestris) is the natural reservoir of T. brevior with prevalences of infection of up to $71.4 \%[4,8,13-15]$. A study carried out in southern Italy did not clarify whether this lungworm is a 
threat for wildcat populations, despite the high prevalence recorded and a negative correlation between $T$. brevior burden and body condition index [13]. Nonetheless, a more recent study has shown that $T$. brevior may actually cause moderate to severe lung lesions in infected F. s. silvestris [14].

Despite an apparent spreading of $T$. brevior in both domestic and wild cats [4, 13-15], no reports of infections with Troglostrongylus spp. in another endangered European wild felid, i.e. the Eurasian lynx ( $\operatorname{Lyn} x \operatorname{lyn} x)$, are available. Indeed, wild $\operatorname{Lyn} x$ spp. are frequently infected with $T$. wilsoni, i.e. the bobcat (Lynx rufus) in several regions of the United States $[16,17]$ and the Canada lynx (Felis canadensis) in northern Ontario [18]. Given the merit to enhance our knowledge on the occurrence and the impact of troglostrongylosis in the European wildlife, this paper describes for the first time pneumonia associated with $T$. brevior in an Eurasian lynx illegally killed in Bosnia and Herzegovina.

\section{Methods}

In February 2014, the carcass of a $c$. 3-year-old male Eurasian lynx was presented for necropsy at the Department of Pathology, Faculty of Veterinary Medicine in Sarajevo. The lynx was shot by poachers in Central Bosnia, in the Fojnica municipality $\left(43^{\circ} 57^{\prime} 344^{\prime \prime} \mathrm{N}, 17^{\circ}\right.$ $\left.54^{\prime} 10 " \mathrm{E}\right)$ on the eastern slopes of the Vranica Mountain. The animal was in a good body condition. Tissue samples from lungs, kidneys, intestine, stomach, liver and spleen, were fixed in $10 \%$ neutral buffered formalin overnight, embedded in paraffin, and cut at 3 to $6 \mu \mathrm{m}$ sections. Deparaffinised sections were stained with hematoxilin and eosin and examined under a light microscope. Tracheal mucosa was scraped and examined under the light microscope. Genomic DNA was extracted from three paraffinembedded lung tissue samples where morulated eggs and embedded nematode first stage larvae were analysed as previously described [19], molecularly examined [20] and the amplicon sequenced. The sequence obtained was aligned using Data Analysis in Molecular Biology and Evolution version 4.5.55 (DAMBE) and compared with those of other nematodes available in the GenBank ${ }^{\circ}$ using the Nucleotide-Nucleotide "Basic Local Alignment Search Tool" (BLAST).

\section{Results}

At necropsy, multifocal, multinodular, consolidated and firm, $\tan$ to grey, areas, occupying the caudal third of both caudal lung lobes were observed (Fig. 1). Multiple 0.5 to $1.5 \mathrm{~cm}$ foci of similar appearance were present on the apical and cardiac lobes. Lung parenchyma was bright-red and a focal perforation with haemorrhage and oedema (bullet wound) was recorded on the caudal acute edge of the left apical lobe. At cut section of the

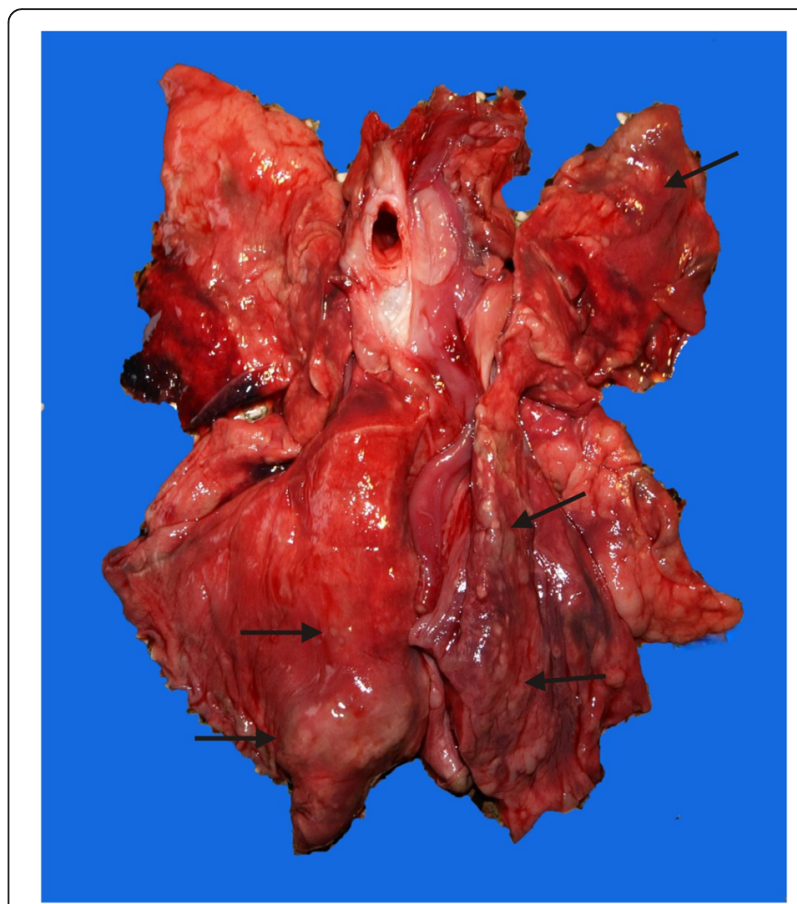

Fig. 1 Multifocal, various sizes grey to tan consolidated areas (arrows) in the parenchyma of the caudal lung lobes of the Eurasian lynx infected with Troglostrongylus brevior

lung, catarrhal fluid was draining from the airways. The tracheal mucosa was covered with a thin layer of red tinged (with blood) mucous material. In the scrapings of the tracheal mucosa multiple first stage larvae (L1) of T. brevior were detected (Fig. 2). No adult parasites were found. No remarkable lesions were observed on other organs.

Histopathology of lung tissue revealed multifocal to coalescing areas, centred on bronchi and bronchioles, and expanded alveoli filled with necrotic debris, degenerated inflammatory cells, mostly neutrophils and macrophages, and multiple cross sections of parasite larvae and thin walled morulated eggs (Fig. 3). The interalveolar septa were necrotic and expanded with moderate infiltrates of neutrophils, macrophages and lesser numbers of eosinophils. At the periphery of these areas, small numbers of multinucleate giant cells were observed. The walls of airways were expanded with moderate infiltrates of mostly neutrophils and eosinophils, and oedema. Multifocal necrotic areas filled with numerous neutrophils and lesser numbers of macrophages and eosinophils were scattered across the remaining parenchyma. There was a mild perivascular and peribronchiolar oedema, mild hyperplasia of media of vessel walls, and multifocal moderate perivascular cuffing of lymphocytes and neutrophils. Homogenous eosinophilic granular masses (thrombosis) were present in the lumen of multiple blood vessels. The paraffin-embedded samples scored molecularly 


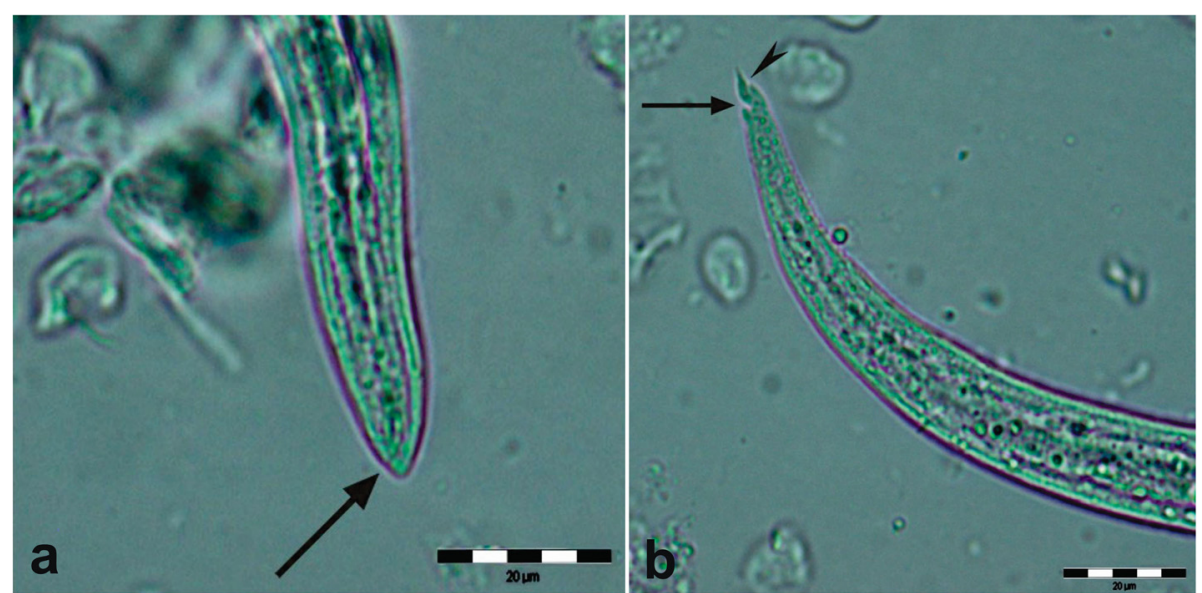

Fig. 2 First stage larva (L1) of Troglostrongylus brevior. a Anterior end with a subterminal opening (arrow). b Pointed tail with a pronounced dorsal (arrow) and shalow ventral (arrowhead) incisures and spines typical of T. brevior. Scale-bars: $20 \mu \mathrm{m}$

positive for T. brevior, with a $100 \%$ homology with the GenBank ${ }^{\circ}$ sequence KF241978.1.

\section{Discussion}

The data provided here represent the first report on the occurrence of $T$. brevior and associated lung pathology in the Eurasian lynx, L. lynx. Interestingly, until now Troglostrongylus spp. has not been detected in several studies of the Eurasian lynx parasite fauna in Europe [21-24], despite these lungworms occur frequently in bobcat and Canada lynx in North America [16-18]. The absence of Troglostrongylus spp. in the European lynx could be related to the use of only copromicroscopic and morphological methods applied in the previous studies, or due to the geographic area of sampling, that could be unsuitable for the biology of this lungworm [21-24]. Thus, the infection might have been misdiagnosed for the cat lungworm Aelurostrongylus abstrusus Railliet, 1898 [25]. In fact, L. lynx was considered a host of $A$. abstrusus [25], based on past copromicroscopic findings [26]. Nevertheless, morphometric data of those L1 identified as A. abstrusus [26] do not fall within the ranges reported for this nematode [6] and could represent a misdiagnosis of a Troglostrongylus spp. [27].

The lesions observed in the lung of the present case are almost identical to those described in a

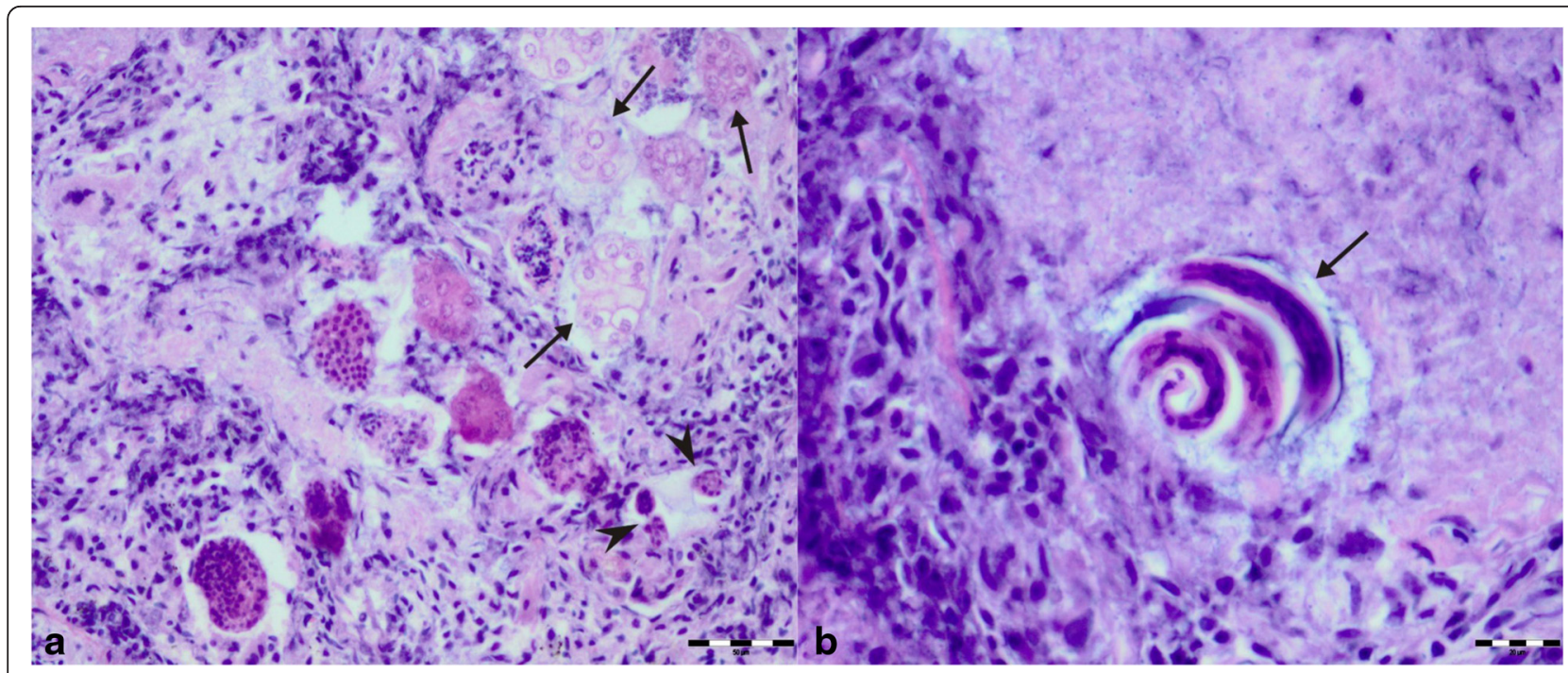

Fig. 3 Histopathology of lung lesions associated with Troglostrongylus brevior infection in an Eurasian lynx (Lynx lynx). a Distended alveolar ducts and bronchioles filled with exudate, degenerated cells and multiple cross sections of parasites (arrowheads) and thin walled morulated eggs (arrows) in the lung of Eurasian lynx infected with Troglostrongylus brevior. b First stage larva (L1) (arrow) of Troglostrongylus brevior in the exudate filled distended alveolar duct of the Eurasian lynx. Hematoxilin and eosin staining. Scale-bars: a, $50 \mu \mathrm{m} ; \mathbf{b}, 20 \mu \mathrm{m}$ 
kitten co-infected with $A$. abstrusus and T. brevior [11]. No adult parasites were observed in the trachea or the bronchi of this lynx. The presence of larvae and morulated eggs in distended alveolar spaces surrounded with eosinophils, neutrophils and macrophages are lesions more attributed to A. abstrusus than to T. brevior. Lesions caused by $T$. brevior and $A$. abstrusus differ in localisation and intensity, as $T$. brevior is larger and mostly localised in the medium-sized and large bronchi and bronchioles, whereas A. abstrusus is smaller and localised in the alveolar ducts and subpleural parenchyma [1]. In cases of mixed infections the overlapping of lesions makes challenging to discern the exact cause of lung damage [11]. A mixed infection by A. abstrusus and T. brevior can be here considered unlikely because of the lack of $A$. abstrusus-typical lesions (e.g. subpleural nodules) and the negative result for A. abstrusus in a very sensitive DNA-based assay [20]. Additionally, a catarrhal bronchitis, as here described, is typical of troglostrongylosis in both domestic $[1,10,11]$ and wild cats [14], and less evident in aelurostrongylosis, where macrophages are often organised in granulomas [28]. In the present case, however, no subpleural nodular aggregates of macrophages were observed. Furthermore, L1s (Fig. 2) detected in the tracheal scrapings have the typical characteristics of $T$. brevior. In any case, the presence of $A$. abstrusus in the animal examined here cannot be ultimately ruled out because the genomic DNA of $A$. abstrusus obtained from parrafin-embedded tissue could have been too fragmented to provide binding sites from specific primers or because A. abstrusus was not present in the three examined samples.

No lesions caused by T. wilsoni have been described in $\operatorname{Lyn} x$ spp., apart from the proteinaceous fluid noted in the lung of one bobcat in Alabama, United States [17]. Although the cause of death of the lynx of the present case was a disgraceful and illegal killing by poachers, the extent of the recorded pneumonia demonstrates the ability of $T$. brevior to cause lesions and to hamper the respiratory system of this wild felid. Hence, further studies are warranted to investigate the occurrence of lungworms in this endangered felid species and if and how they may represent a threat for host's health and welfare. In fact, as the domestic cat, the Eurasian lynx could be affected by an overflow of pathogenic lungworms that are usually harboured by wildcats [4].

\section{Conclusions}

This paper describes for the first time infection with $T$. brevior in an European lynx and the associated lung pathology. Given its pathogenic potential and the lack of data on Troglostrongylus spp. in lynx populations, the occurrence and impact of lungworms on Eurasian lynx health and welfare should be further investigated. Moreover, further studies are necessary to better elucidate the epizootiology of $T$. brevior in felids other than Felis spp., in order to understand whether the infection in the European lynx is due to bridging infections $[2,4]$ or, as indicated for the European wildcat $[2,4,13]$, it represents another reservoir of troglostrongylosis.

\section{Competing interests}

The authors declare that they have no competing interests.

\section{Authors' contributions}

AA, AH and DT drafted and wrote the manuscript; AA performed the necropsy and histopathology; DT and ADC performed the molecular examinations and critically revised the manuscript; GGD and MK revised the manuscript. All authors read and approved the final manuscript.

\section{Acknowledgements}

We would like to thank Alma Jahić, Faculty of Veterinary Medicine, Sarajevo for technical assistance for histopathology.

\section{Author details}

${ }^{1}$ Department of Pathology, Faculty of Veterinary Medicine, University of Sarajevo, Sarajevo, Bosnia and Herzegovina. ${ }^{2}$ Teaching Veterinary Hospital, Faculty of Veterinary Medicine, University of Teramo, Teramo, Italy. ${ }^{3}$ Institute of Parasitology, Department of Pathobiology, University of Veterinary Medicine Vienna, Veterinaerplatz 1, 1210 Vienna, Austria.

Received: 9 November 2015 Accepted: 16 December 2015

Published online: 21 December 2015

\section{References}

1. Brianti E, Giannetto S, Dantas-Torres F, Otranto D. Lungworm of the genus Troglostrongylus (Strongylida: Crenosomatidae): Neglected parasites for domestic cats. Vet Parasitol. 2014;202:104-12.

2. Traversa D, Di Cesare A. Feline lungworms: what a dilemma. Trends Parasitol. 2013;29:423-30

3. Brianti E, Gaglio G, Napoli E, Falsone L, Giannetto S, Latrofa MS, et al. Evidence for direct transmission of the cat lungworm Troglostrongylus brevior (Strongylida: Crenosomatidae). Parasitol. 2013;140:821-4.

4. Di Cesare A, Veronesi F, Traversa D. Felid lungworms and heartworms in Italy: more questions than answers? Trends Parasitol. 2015; in press.

5. Jefferies R, Vrhovec MG, Wallner N, Catalan DR. Aelurostrongylus abstrusus and Troglostrongylus sp. (Nematoda: Metastrongyloidea) infections in cats inhabiting Ibiza, Spain. Vet Parasitol. 2010;173:344-8.

6. Brianti E, Gaglio G, Giannetto S, Annoscia G, Latrofa MS, Dantas-Torres F, et al. Troglostrongylus brevior and Troglostrongylus subcrenatus (Strongylida: (renosomatidae) as agents of broncho-pulmonary infestation in domestic cats. Parasit Vectors. 2012;5:178.

7. Tamponi C, Varcasia A, Brianti E, Pipia AP, Frau V, Pinna Parpaglia ML, et al. New insights on metastrongyloid lungworms infecting cats of Sardinia, Italy. Vet Parasitol. 2014:203:222-6.

8. Diakou A, Di Cesare A, Aeriniotaki T, Traversa D. First report of Troglostrongylus brevior in a kitten in Greece. Parasitol Res. 2014;113:3895-8.

9. Di Cesare A, Frangipane di Regalbono A, Tessarin C, Seghetti M, lorio R, Simonato $G$, et al. Mixed infection by Aelurostrongylus abstrusus and Troglostrongylus brevior in kittens from the same litter in Italy. Parasitol Res. 2014;113:613-8.

10. Giannelli A, Passantino G, Ramos RAN, Lo Presti G, Lia RP, Brianti E, et al. Pathological and histological findings associated with the feline lungworm Troglostrongylus brevior. Vet Parasitol. 2014;204:416-9.

11. Traversa D, Romanucci M, Di Cesare A, Malatesta D, Cassini R, lorio R, et al. Gross and histopathological changes associated with Aelurostrongylus abstrusus and Troglostrongylus brevior in a kitten. Vet Parasitol. 2014;201:158-62.

12. Di Cesare A, Veronesi F, Grillotti E, Manzocchi S, Perrucci S, Beraldo P, et al. Respiratory nematodes in cat populations of Italy. Parasitol Res. 2015:114:4463-9.

13. Falsone L, Brianti E, Gaglio G, Napoli E, Anile S, Mallia E, et al. The European wildcats (Felis silvestris silvestris) as reservoir hosts of Troglostrongylus brevior (Strongylida: Crenosomatidae) lungworms. Vet Parasitol. 2014;205:193-8. 
14. Veronesi F, Traversa D, Lepri E, Morganti G, Vercillo F, Grelli D, et al. Occurrence of lungworms in European wildcats (Felis silvestris silvestris) of central Italy. J Wild Dis. 2015; in press.

15. Beraldo P, Massimo M, Pascotto E. Analysis of the helminthofauna of European wild cat in Friuli Venezia Giulia. In: Proceedings of XXVIII Nationa Conference of the Italian Society of Parasitology, Mappe Parassitologiche ed. University of Naples Federico II. Naples, Italy; 2014. p. 225.

16. Sarmiento L, Stough BD. Troglostrongylus wilsoni (Stough, 1953) n. comb. (Nematoda: Metastrongylidae) from the lungs of the bobcat, Lynx rufus rufus. J Parasitol. 1956;42:45-8.

17. Reichard MV, Caudell DL, Kocan AA. Survey of Helminth Lung Parasites of Bobcats (Lynx rufus) from Alabama, Kansas, New Mexico, Oklahoma, and Virginia, U.S.A. Comp Parasitol. 2004;71:88-90.

18. Smith JD, Addison EM, Joachim DG, Smith LM, Quinn NWS. Helminth parasites of Canada lynx (Felis canadensis) from northern Ontario. Can J Zool. 1986;64:358-64.

19. Di Cesare A, Di Francesco G, Frangipane di Regalbono A, Eleni C, De Liberato C, Marruchella G, et al. Retrospective study on the occurrence of the feline lungworms Aelurostrongylus abstrusus and Troglostrongylus spp. in endemic areas of Italy. Vet J. 2015;203:233-8.

20. Di Cesare A, Veronesi F, Frangipanedi Regalbono A, lorio R, Traversa D. Novel molecular assay for the simultaneous identification of neglected lungworms and heartworms affecting cats. J Clin Microbiol. 2015:53:3009-13.

21. Schmidt-Posthaus H, Breitenmoser-Wursten C, Posthaus H, Bacciarini L, Breitenmoser U. Causes of mortality in reintroduced Eurasian lynx in Switzerland. J Wild Dis. 2002;38:84-92.

22. Valdmann H, Moks E, Talvik H. Helminth fauna of Eurasian lynx (Lynx lynx) in Estonia. J Wildl Dis. 2004;40:356-60.

23. Szczesna J, Popiołek M, Schmidt K, Kowalczyk R. Coprological Study on helminth fauna in Eurasian lynx (Lynx lynx) from the Białowieza Primeval Forest in Eastern Poland. J Parasitol. 2008;94:981-84.

24. Deksne G, Laakkonen J, Nareaho A, Jokelainen P, Holmala K, Kojola I, et al. Endoparasites of the Eurasian lynx (Lynx lynx) in Finland. J Parasitol. 2013:99:229-34

25. Otranto D, Brianti E, Dantas-Torres F. Troglostrongylus brevior and a nonexistent 'dilemma'. Trends Parasitol. 2013:29:517-8.

26. Szczęsna J, Popiołek M, Schmidt K, Kowalczyk R. The first record of Aelurostrongylus abstrusus (Angistrongylidae: Nematoda) in Eurasian lynx (Lynx lynx L.) from Poland based on fecal analysis. Wiad Parazytol. 2006;52:321-2

27. Traversa D. Response to Otranto et al. Lungworms in domestic and wild felids: dilemmas still persisting. Trends Parasitol. 2014;30:53-4.

28. Schnyder M, Di Cesare A, Basso W, Guscetti F, Riond B, Glaus T, et al. Clinical, laboratory and pathological findings in cats experimentally infected with Aelurostrongylus abstrusus. Parasitol Res. 2014;113:1425-33.

\section{Submit your next manuscript to BioMed Central and we will help you at every step:}

- We accept pre-submission inquiries

- Our selector tool helps you to find the most relevant journal

- We provide round the clock customer support

- Convenient online submission

- Thorough peer review

- Inclusion in PubMed and all major indexing services

- Maximum visibility for your research

Submit your manuscript at www.biomedcentral.com/submit

) Biomed Central 